\title{
Expansion of the Confluent Hypergeometric Function in Series of Bessel Functions
}

\author{
By Yudell L. Luke
}

\begin{abstract}
An expansion of the confluent hypergeometric function $\Phi(a, c, z)$ in series of functions of the same kind has been given by Buchholz [1]. By specialization of some quantities, there is obtained an expansion in series of modified Bessel functions of the first kind, $I_{v}(z)$, where $\nu$ depends on the parameter $a$. Tricomi $[2,3]$ has developed two expansions of similar type where both the order and argument of the Bessel functions depend on the parameters $a$ and $c$. In the present paper, we derive an expansion in series of Bessel functions of integral order whose argument is independent of $a$ and $c$. Our expansion is advantageous for many purposes of computation since the parameters and variable of $\Phi(a, c, z)$ appear in separated form. Also, for desk calculation, extensive tables of $I_{n}(z)$ are available, while for automatic computation Bessel functions are easy to generate [4].

Special cases of the confluent function, such as the incomplete gamma function, are also studied. For the class of transcendents known as the error functions, including the Fresnel integrals, it is shown that our expansion coincides with that of Buchholz [1]. By specializing a parameter and passing to a limit, we derive expansions for the exponential integral and related functions. Other expansions for the error and exponential integrals are derived on altogether different bases. Finally, some numerical examples are presented to manifest the efficiency of our formulas.
\end{abstract}

1. Representation of the Confluent Hypergeometric Function in Series of Bessel Functions of Integral Order. The confluent hypergeometric function is defined by the series

$$
\begin{aligned}
\Phi(a, c, z) & \equiv{ }_{1} F_{1}(a ; c ; z)=\sum_{k=0}^{\infty} \frac{(a)_{k} z^{k}}{(c)_{k} k !}, \\
(a)_{k} & =\frac{\Gamma(a+k)}{\Gamma(a)},
\end{aligned}
$$

where $\Gamma(z)$ is the gamma function. We also have the integral representation

$$
\Phi(a, c, z)=\frac{\Gamma(c)}{\Gamma(a) \Gamma(c-a)} \int_{0}^{1} e^{z t} t^{a-1}(1-t)^{c-a-1} d t, \quad R(c)>R(a)>0,
$$

and Kummer's formula

$$
\Phi(a, c, z)=e^{z} \Phi(c-a, c,-z) .
$$

For these and other properties of the confluent function, see [5].

Let $t=\sin ^{2} \theta$ in the integrand of (12). Using the Jacobi expansion [6]

$$
e^{(z / 2) \cos 2 \theta}=\sum_{k=0}^{\infty} \epsilon_{k} \cos 2 k \theta I_{k}(z / 2), \quad \epsilon_{0}=1, \quad \epsilon_{k}=2, \quad k>0,
$$

Received May 26, 1959. This research was supported by the United States Air Force through the Air Force Office of Scientific Research of the Air Research and Development Command. 
(1.2) becomes

$$
\begin{aligned}
\Phi(a, c, z)=\frac{2 \Gamma(c)}{\Gamma(a) \Gamma(c-a)} e^{z / 2} \sum_{k=0}^{\infty}(-1)^{k} \epsilon_{k} I_{k}(z / 2) \\
\cdot \int_{0}^{\frac{\pi}{2}} \cos 2 k \theta(\sin \theta)^{2 a-1}(\cos \theta)^{2 c-2 a-1} d \theta .
\end{aligned}
$$

Since

$$
\cos 2 k \theta=(-1)^{k} \sum_{m=0}^{k} \frac{(-1)^{m}\left(\begin{array}{l}
k \\
m
\end{array}\right)(k)_{m}(\cos \theta)^{2 m}}{(1 / 2)_{m}}
$$

where $\left(\begin{array}{l}k \\ m\end{array}\right)$ is the binomial coefficient, and

$$
\int_{0}^{\frac{\pi}{2}}(\sin \theta)^{2 x-1}(\cos \theta)^{2 y-1} d \theta=\frac{1}{2} \frac{\Gamma(x) \Gamma(y)}{\Gamma(x+y)}, \quad R(x)>0, \quad R(y)>0,
$$

we find that

$$
\Phi(a, c, z)=e^{z / 2} \sum_{k=0}^{\infty}(-1)^{k} \epsilon_{k} R_{k}(a, c) I_{k}(z / 2),
$$

where $R_{k}(a, c)$ is conveniently expressed in hypergeometric form [7] as

$$
R_{k}(a, c)={ }_{3} F_{2}\left(-k, k, a ; c, \frac{1}{2} ; 1\right) .
$$

An alternative expression for $R_{k}(a, c)$ is readily reduced from a result in [8]. We have

$$
\begin{aligned}
& \frac{\Gamma(a) \Gamma(c-a)}{\Gamma(c)} R_{k}(a, c)=G_{k}(a, c)+(-1)^{k} G_{k}(c-a, c), \\
& G_{k}(a, c)=\frac{(-1)^{k} \cos (c-a) \pi \Gamma(k-c+1) \Gamma(2 c-2 a)}{2^{2 c-2 a-1} \Gamma(k+1+c-2 a)} \\
& \cdot{ }_{2} F_{1}\left(1-2 a, 2 c-2 a ; k+1+c-2 a ; \frac{1}{2}\right) .
\end{aligned}
$$

If $a$ and $c$ are fixed and $k$ is sufficiently large, then the ${ }_{2} F_{1}$ 's in (1.10) are of order unity and

$$
\begin{aligned}
& \frac{\Gamma(a) \Gamma(c-a)}{\Gamma(c)} R_{k}(a, c) \approx \frac{(-1)^{k} \cos (c-a) \pi \Gamma(2 c-2 a) k^{2 a-2 c}}{2^{2 c-2 a-1}} \\
&+\frac{\cos a \pi \Gamma(2 a) k^{-2 a}}{2^{2 a-1}}
\end{aligned}
$$

Here we have used the fact that for fixed $\alpha$ and $\beta$,

$$
\frac{\Gamma(k+\alpha)}{\Gamma(k+\beta)} \approx k^{\alpha-\beta}, \quad k \text { large }
$$


Again, if $z$ is fixed and $k$ is large, then

$$
I_{k}(z) \approx \frac{(z / 2)^{k}}{k !}
$$

It follows that (1.8) converges like

$$
\sum_{k=1}^{\infty} \frac{\left(A k^{2 a-2 c}+B k^{-2 a}\right) z^{k}}{2^{2 k} k !}
$$

$A$ and $B$ are constants. A similar argument shows that (1.1) converges like

$$
\sum_{k=1}^{\infty} \frac{k^{a-c} z^{k}}{k !}
$$

and so (1.8) converges more rapidly than (1.1).

Some general properties of $R_{k}(a, c)$ are next of interest. The combination of (1.3) and (1.8) shows that

$$
R_{k}(a, c)=(-1)^{k} R_{k}(c-a, c) ; \quad R_{k}(a, a)=(-1)^{k} .
$$

Further relations follow by applying known contiguous relations of $\Phi(a, c, z)$ to (1.8). For example, since

$$
\frac{d}{d z} \Phi(a, c, z)=\frac{a z}{c} \Phi(a+1, c+1, z),
$$

it follows that

$$
R_{k}(a+1, c+1)=-\frac{c}{4 a}\left[R_{k+1}(a, c)-2 R_{k}(a, c)+R_{k-1}(a, c)\right]
$$

and

$$
(a-c+1) R_{k}(a, c)-a R_{k}(a+1, c)+(c-1) R_{k}(a, c-1)=0,
$$

results from a contiguous formula of the form (1.19) with $R_{k}(a, c)$ replaced by $\Phi(a, c, z)$. To obtain a pure recursion formula for $R_{k}(a, c)$, apply the differential equation satisfied by $\Phi(a, c, z)$ to (1.8). Employ the difference-differential properties of $I_{n}(z)$ and equate to zero the coefficient of $I_{k}(z)$. We get

$$
(k+c) R_{k+1}(a, c)=2(c-2 a) R_{k}(a, c)+(k-c) R_{k-1}(a, c) .
$$

For certain values of the parameters, the coefficients $R_{k}(a, c)$ can be expressed in simple form. Thus from (1.10),

$$
R_{k}\left(\frac{1}{2}, c\right)=\frac{[\Gamma(c)]^{2}}{\Gamma(c+k) \Gamma(c-k)}, \quad c \neq 0,-1,-2, \cdots, R(c)>0 .
$$

This also follows from (1.9) and the known expression for $a_{2} F_{1}$ with unit argument. If $c=2 a$, then from (1.9) and (1.20), $R_{k}(a, 2 a)$ is zero if $k$ is odd, and

$$
R_{2 k}(a, 2 a)=\frac{\left(\frac{1}{2}-a\right)_{k}}{\left(\frac{1}{2}+a\right)_{k}}, \quad a \neq-\frac{1}{2},-\frac{3}{2},-\frac{5}{2} \cdots
$$

Since

$$
\Phi\left(\nu+\frac{1}{2}, 2 \nu+1,-z\right)=\Gamma(\nu+1)(z / 4)^{-\nu} e^{-z / 2} I_{\nu}(z / 2),
$$


it follows that

$$
I_{\nu}(z)=(z / 2)^{\nu} \Gamma(\nu+1) \sum_{k=0}^{\infty} \frac{(-1)^{k} \epsilon_{k} I_{2 k}(z)}{\Gamma(\nu-k+1) \Gamma(\nu+k+1)}
$$

provided $\nu$ is not a negative integer The latter is a special case of a known result [9].

2. The Incomplete Gamma Function. If $c=a+1$, we write

$$
\begin{gathered}
\gamma(a, z)=a^{-1} z^{a} e^{-z} \Phi(1,1+a, z)=a^{-1} z^{a} \Phi(a, a+1,-z), \\
\gamma(a, z)=\int_{0}^{z} e^{-t} t^{a-1} d t=\Gamma(a)-\int_{z}^{\infty} e^{-t} t^{a-1} d t, \quad R(a)>0 .
\end{gathered}
$$

Thus

$$
\begin{aligned}
R_{k}(a) \equiv R_{k}(a, a+1)= & { }_{3} F_{2}\left(-k, k, a ; a+1, \frac{1}{2} ; 1\right) \\
= & (-1)_{3}^{k} F_{2}\left(-k, k, 1 ; a+1, \frac{1}{2} ; 1\right) \\
= & \frac{-(-1)^{k} a}{2(k-a)(k-a+1)}{ }_{2} F_{1}\left(1-2 a, 2 ; k-a+2 ; \frac{1}{2}\right) \\
& +a \frac{\cos a \pi \Gamma(2 a) k \Gamma(k-a)}{2^{2 a-1} \Gamma(k+a+1)},
\end{aligned}
$$

and

$$
\begin{gathered}
(k+a+1) R_{k+1}(a)=2(1-a) R_{k}(a)+(k-a-1) R_{k-1}(a), \\
R_{k}(a+1)=-\frac{(a+1)}{4 a}\left[R_{k+1}(a)-2 R_{k}(a)+R_{k-1}(a)\right] .
\end{gathered}
$$

If $a=\frac{1}{2}$, we deduce the following expressions for the error function and related functions.

$$
\begin{gathered}
\operatorname{Erf} z=\int_{0}^{z} e^{-t^{2}} d t=-z \sum_{k=0}^{\infty} \frac{(-1)^{k} \epsilon_{k} e^{-z^{2} / 2} I_{k}\left(z^{2} / 2\right)}{4 k^{2}-1} \\
e^{-z^{2}} \text { Erfi } z=e^{-z^{2}} \int_{0}^{z} e^{t^{2}} d t=-z \sum_{k=0}^{\infty} \frac{\epsilon_{k} e^{-z^{2} / 2} I_{k}\left(z^{2} / 2\right)}{4 k^{2}-1} . \\
(2 \pi)^{\frac{1}{2}}[C(z)+i S(z)]=\int_{0}^{z} t^{-\frac{1}{2}} e^{i t} d t=-2 z^{\frac{3}{k}} e^{i z / 2} \sum_{k=0}^{\infty} \frac{\epsilon_{k} i^{k} J_{k}(z / 2)}{4 k^{2}-1} .
\end{gathered}
$$

A more general expansion for the error function can be found as follows. Now

$$
\int_{0}^{z \sin \theta} e^{-t^{2}} d t=z e^{-z^{2} / 2} \int_{0}^{\theta} e^{\left(z^{2} / 2\right) \cos 2 t} \cos t d t
$$

and using (1.4), we obtain

(2.11) $\operatorname{Erf}(z \sin \theta)=\frac{z}{2} \sum_{k=0}^{\infty} \epsilon_{k}\left\{\frac{\sin (2 k+1) \theta}{2 k+1}+\frac{\sin (2 k-1) \theta}{2 k-1}\right\} e^{-z^{2} / 2} I_{k}\left(z^{2} / 2\right)$, 
and from the latter follows the interesting representation

$$
\int_{0}^{\frac{\pi}{2}} \operatorname{Erf}(z \sin \theta) \sin (2 k+1) \theta d \theta=\frac{\pi z}{4(2 k+1)} e^{-z^{2} / 2}\left[I_{k}\left(z^{2} / 2\right)+I_{k+1}\left(z^{2} / 2\right)\right] .
$$

3. The Exponential Integral. This is a limiting form of the incomplete gamma function. From (2.2), we have

$$
\int_{z}^{\infty} t^{-1} e^{-t} d t=\lim _{a \rightarrow 0}\{\Gamma(a)-\gamma(a, z)\} .
$$

Another useful form is

$$
\int_{0}^{z} t^{-1}\left(1-e^{-t}\right) d t=\lim _{a \rightarrow 0}\left\{a^{-1} z^{a}-\gamma(a, z)\right\} .
$$

Using (1.4) and (2.3), we write

$$
e^{z / 2} z^{-a} \int_{z}^{\infty} e^{-u} u^{a-1} d u=\left\{\frac{\Gamma(a+1) z^{-a}-1}{a}\right\} e^{z / 2}+2 \sum_{k=1}^{\infty}\left\{\frac{1-R_{k}(a)}{a}\right\} I_{k}(z / 2) .
$$

Application of L'Hospital's theorem gives

$$
\lim _{a \rightarrow 0}\left\{\frac{\Gamma(a+1) z^{-a}-1}{a}\right\}=-(\gamma+\ln z)
$$

where $\gamma$ is Euler's constant. Define

$$
f_{k}=\lim _{a \rightarrow 0} \frac{1-R_{k}(a)}{a}=-\sum_{m=1}^{k} \frac{(-1)^{m}\left(\begin{array}{l}
k \\
m
\end{array}\right)(k)_{m}}{\left(\frac{1}{2}\right)_{m} m} .
$$

Then

$$
\begin{aligned}
-E i(-z)+(\gamma+\ln z) & =\int_{z}^{\infty} t^{-1} e^{-t} d t+(\gamma+\ln z) \\
& =\int_{0}^{z} t^{-1}\left(1-e^{-t}\right) d t=2 \sum_{k=1}^{\infty} f_{k} e^{-z / 2} I_{k}(z / 2) .
\end{aligned}
$$

In view of (2.5) and (3.5), we have the recurrence formula

$$
(k+1) f_{k+1}=2 f_{k}+(k-1) f_{k-1}+4,
$$

and by induction or otherwise, we can prove that

$$
\left\{\begin{array}{l}
f_{k+1}=f_{k}+\frac{1}{k}\left\{1-(-1)^{k}\right\}+\frac{1}{k+1}\left\{1+(-1)^{k}\right\}, \quad f_{0}=0, f_{1}=2 \\
f_{2 k}=4\left(1+\frac{1}{3}+\cdots+\frac{1}{2 k-1}\right) \\
f_{2 k+1}=f_{2 k}+\frac{2}{2 k+1}, \quad f_{2 k+2}=f_{2 k+1}+\frac{2}{2 k+1}
\end{array}\right.
$$

Employing the theory of the $\psi(z)$ function, the logarithmic derivative of the 
gamma function [10], we find that

$$
\lim _{k \rightarrow \infty}\left(f_{k}-2 \ln 2 k\right)=2 \gamma,
$$

and so (3.6) converges like

$$
\sum_{k=1}^{\infty} u_{k} z^{k}, \quad u_{k}=(\ln k) / 2^{2 k} k !
$$

An alternative form of (3.6) is

$$
\left\{\begin{array}{l}
\int_{0}^{z} t^{-1}\left(1-e^{-t}\right) d t=2\left[1-e^{-z / 2} I_{0}(z / 2)\right]+4 \sum_{k=0}^{\infty} g_{k+2} e^{-z / 2} I_{k+2}(z / 2) \\
g_{k}=\frac{1}{2} f_{k}-1
\end{array}\right.
$$

Now

$$
E i(z)=\int_{-\infty}^{z} t^{-1} e^{t} d t=E i\left(z e^{ \pm i \pi}\right) \mp i \pi
$$

and so

$$
\begin{aligned}
e^{-z} \int_{-\infty}^{z} t^{-1} e^{t} d t=e^{-z}(\gamma+\ln z)+2\left[e^{-z / 2} I_{0}(z / 2)-e^{-z}\right] \\
-4 \sum_{k=0}^{\infty}(-1)^{k} g_{2 k+2} e^{-z / 2} I_{k+2}(z / 2)
\end{aligned}
$$

We also have

$$
\begin{aligned}
\int_{0}^{z} t^{-1}\left(1-e^{-i t}\right) d t & =(\gamma+\ln z)-C i(z)+i \operatorname{Si}(z) \\
& =2 e^{-i z / 2} \sum_{k=1}^{\infty} f_{k} i^{k} J_{k}(z / 2)
\end{aligned}
$$

4. A Second Expansion and Special Cases. Buchholz [1] has given the expansion

$$
\begin{aligned}
& \Phi(a, c, z)=e^{z / 2}(z / 4)^{\frac{1-a}{2}} \Gamma\left(a-\frac{1}{2}\right) \\
& \cdot \sum_{k=0}^{\infty} \frac{\left(k+a-\frac{1}{2}\right)(2 a-1)_{k}(2 a-c)_{k}}{k !(c)_{k}} I_{k+a-\frac{1}{2}}(z / 2), \quad R(a)>0,
\end{aligned}
$$

which coalesces with (1.8) when $a=\frac{1}{2}$. If $a, c$ and $z$ are fixed, then an analysis similar to (1.11)-(1.14) shows that (4.1) converges like

$$
\sum_{k=1}^{\infty} \frac{k^{3 a-2 c-1 / 2} z^{k}}{2^{2 k} k !},
$$

and from this point of view, the convergence of (1.8) and (4.1) are nearly alike when $R(c-a)>0$.

We now deduce from (4.1) series expansions for the exponential integral and related functions. The method of proof is akin to that in the previous section. If in (4.1), we put $a=c$, there follows a representation for $e^{z / 2} \operatorname{since} \Phi(a, a, z)=e^{z}$. Use this and (4.1) with $c=a+1$ and $z$ replaced by $-z$. Then after the manner 
of $(3.3)$, we have

$$
e^{z / 2} \int_{z}^{\infty} t^{a-1} e^{-t} d t=(\pi z)^{1 / 2} \sum_{k=0}^{\infty} h_{k}(a) I_{k+a-1 / 2}(z / 2),
$$

and employing the duplication formula for gamma functions, the elements $h_{k}(a)$ take the form

$$
\begin{aligned}
h_{k}(a)=\frac{2^{k+2 n-1}(k+a-1 / 2) \Gamma\left(\frac{k-1}{2}+a\right) \Gamma\left(\frac{k}{2}+a\right)}{\Gamma\left(\frac{1}{2}\right) k !} \\
\cdot\left\{z^{-a}-\frac{(-1)^{k}}{\Gamma(a-1)(k+a)(k+a-1)}\right\} .
\end{aligned}
$$

For $k=0$ and 1, application of L'Hospital's theorem gives

$$
h_{0}(0)=-\frac{1}{2}(\gamma+\ln z), \quad h_{1}(0)=h_{0}(0)+1 .
$$

Otherwise,

$$
h_{k}(0)=\frac{2 k-1}{k(k-1)}, \quad k>1
$$

Thus

$$
\begin{array}{r}
-E i(-z)+(\gamma+\ln z)=\int_{z}^{\infty} t^{-1} e^{-t} d t+(\gamma+\ln z)=\int_{0}^{z} t^{-1}\left(1-e^{-t}\right) d t \\
=(\pi z)^{1 / 2} e^{-z / 2} I_{1 / 2}(z / 2)+(\pi z)^{1 / 2} \sum_{k=0}^{\infty} \frac{(2 k+3)}{(k+1)(k+2)} e^{-z / 2} I_{k+3 / 2}(z / 2)
\end{array}
$$

and forms for the related functions follow readily enough after the manner of (3.12)-(3.14). By a familiar argument, (4.7) converges like

$$
\sum_{k=1}^{\infty} v_{k} z^{k}, \quad v_{k}=\left[2^{2 k} k \Gamma\left(k+\frac{1}{2}\right)\right]^{-1},
$$

and so (4.7) converges more rapidly than (3.6). However, the difference is not great since $u_{k} / v_{k} \sim k^{\frac{1}{2}} \ln k($ see $(3.10))$.

5. Further Expansions. The following representation is due to Tricomi $[2,3]$.

$$
\begin{gathered}
\Phi(a, c, z)=\Gamma(c) e^{h z} \sum_{k=0}^{\infty} A_{k}(a, c, h) z^{k} E_{c+k-1}(-a z), \quad h \geqq 0, \\
E_{\nu}(z)=z^{-\nu / 2} J_{\nu}\left(2 z^{\frac{1}{2}}\right), \quad E_{\nu}(-z)=z^{-v / 2} I_{\nu}\left(2 z^{\frac{1}{2}}\right),
\end{gathered}
$$

where the coefficients $A_{k}(a, c, h)$ are defined by the generating function

$$
\sum_{k=0}^{\infty} A_{k}(a, c, h) z^{k}=e^{-a z}[1+(h-1) z]^{-a}(1+h z)^{a-c}, \quad|z|<1,
$$

and satisfy the recurrence system

$$
\begin{aligned}
& (k+1) A_{k+1}=[(1-2 h) k-h c] A_{k} \\
& +[a(1-2 h)-h(h-1)(c+k-1)] A_{k-1}-h(h-1) A_{k-2}, \\
& A_{0}=1, \quad A_{1}=-h c, \quad A_{2}=\frac{1}{2} h^{2} c(c+1)+a\left(\frac{1}{2}-h\right) .
\end{aligned}
$$


A second expansion given by Tricomi $[2,3]$ is

$$
\Phi(a, c, z)=\Gamma(c) e^{z / 2} \sum_{k=0}^{\infty} A_{k}^{*}(K, c / 2)(z / 2)^{k} E_{c+k-1}(K z), \quad K=\frac{c}{2}-a
$$

where

$$
\begin{gathered}
\sum_{k=0}^{\infty} A_{k}^{*}(K, c / 2) z^{k}=e^{2 K z}(1-z)^{K-c / 2}(1+z)^{-K-c / 2}, \quad|z|<1, \\
(k+1) A_{k+1}^{*}=(k+c-1) A_{k-1}^{*}-2 K A_{k-2}^{*}, \\
A_{0}{ }^{*}=1, \quad A_{1}{ }^{*}=0, \quad A_{2}^{*}=\frac{c}{2} .
\end{gathered}
$$

Both (5.1) and (5.5) are useful to study the behavior of $\Phi$ when the parameters are large [11], but are not too suitable for many purposes of computation, since the order and argument of the Bessel functions depend on the parameters $a$ and $c$.

In (5.1), put $a=1$, replace $c$ by $a+1$ and set $h=0$. Then

$$
\gamma(a, z)=\Gamma(a) e^{-z} z^{a / 2} \sum_{k=0}^{\infty} e_{k}(-1) z^{k / 2} I_{k+a}\left(2 z^{1 / 2}\right), \quad e_{k}(-1)=\sum_{m=0}^{k} \frac{(-1)^{m}}{m !}
$$

follows from (2.1) and (5.3). The method of proof surrounding (1.11)-(1.14) shows that (5.8) converges like $\sum_{k=0}^{\infty} z^{k} / \Gamma(k+a+1)$ which is the same as (1.1) for the above selection of parameters.

6. Further Expansions for the Error and Exponential Integrals. We start with the representation

$$
\begin{aligned}
& \int_{0}^{z} t^{\mu} I_{\nu}(t) d t=\frac{z^{\mu} \Gamma\left(\frac{\nu+\mu+1}{2}\right)}{\Gamma\left(\frac{\nu-\mu+1}{2}\right)} \\
& \cdot \sum_{k=0}^{\infty}(-1)^{k} \frac{(\nu+2 k+1) \Gamma\left(\frac{\nu-\mu+1}{2}+k\right)}{\Gamma\left(\frac{\nu+\mu+3}{2}+k\right)} I_{\nu+2 k+1}(z), \quad R(\mu+\nu+1)>0
\end{aligned}
$$

which is readily verified by differentiation. Put $\mu=0$, and consider the formulas when $\nu=\frac{1}{2}$ and $\nu=-\frac{1}{2}$. Then by subtraction and addition, we get

$$
\operatorname{Erf} z=(\pi / 2)^{\frac{1}{2}} \sum_{k=1}^{\infty}(-1)^{[k / 2]} I_{k-\frac{1}{2}}\left(z^{2}\right)
$$

$$
\text { Erfi } z=(\pi / 2)^{\frac{1}{2}} \sum_{k=0}^{\infty}(-1)^{[k / 2]} I_{k+\frac{1}{2}}\left(z^{2}\right)
$$

Here $[k / 2]$ is the largest integer, including zero, contained in $k / 2$. The latter two equations were also verified by Tricomi [3] by means of the Laplace transform*.

* There is a typographical error in [12] for (6.2). There the summation should start with $n=1, \operatorname{not} n=0$. 
Another representation which follows from (5.8) is

$$
\operatorname{Erf} z=\frac{1}{2}(\pi z)^{\frac{1}{2}} e^{-z^{2}} \sum_{k=0}^{\infty} e_{k}(-1) z^{k} I_{k+\frac{1}{3}}(2 z) .
$$

By a familiar argument, the convergence of (2.7) is superior to that of (6.2)-(6.4).

To obtain another expansion for functions related to the exponential integral, it is convenient to again use (6.1) with $z$ replaced by $i z$. The ensuing formula is of the same type as $(6.1)$ with $I_{\nu}(z)$ replaced by $J_{\nu}(z)$ and the factor $(-1)^{k}$ omitted behind the summation sign. Now put $\mu=-\frac{1}{2}$ and $\nu=\frac{1}{2}$. Then for the sin integral, we have

$$
\int_{0}^{z} \frac{\sin t}{t} d t=\left(\frac{\pi}{2 z}\right)^{1 / 2} \sum_{k=0}^{\infty} \frac{(4 k+3) k !}{\left(\frac{8}{2}\right)_{k}} J_{2 k+3 / 2}(z)
$$

Similarly, with $\nu=-\frac{1}{2}$, we can form an expression for

$$
\begin{gathered}
\int_{0}^{z} t^{\mu-1 / 2}(1-\cos t) d t . \quad \text { Let } \mu \rightarrow-\frac{1}{2} ; \quad \text { then } \\
\int_{0}^{z} t^{-1}(1-\cos t) d t=\frac{1}{2}\left(\frac{\pi}{2 z}\right)^{\frac{1}{2}} \sum_{k=1}^{\infty} \frac{(4 k+1)\left(\frac{1}{2}\right)_{k}}{k !} \\
.\left\{\psi(k+1)-\psi(1)+\psi\left(k+\frac{1}{2}\right)-\psi\left(\frac{1}{2}\right)\right\} J_{2 k+1}(z) .
\end{gathered}
$$

Our previous analysis shows that for $z$ fixed and $k$ large, the ratio of the $k$ th term in the expansion (6.6) to the $k$ th term of the Taylor series expansion for the cosine integral is proportional to $k(\ln k) / 2^{2 k}$. Similarly for $(6.5)$, the ratio is proportional to $k / 2^{2 k}$. If $(3.6)$ is compared with its corresponding Taylor series representation in like fashion, we again obtain the former ratio. Thus the proper combination of (6.5) and (6.6) gives about the same convergence as (.3.6) and (4.7).

As a remark aside, using (6.1), we can produce

$$
\begin{aligned}
& \lim _{\mu \rightarrow-1} \int_{0}^{z} t^{\mu}\left[1-J_{0}(t)\right] d t \\
& \quad=\int_{0}^{z} t^{-1}\left[1-J_{0}(t)\right] d t=(\gamma+\ln z / 2)+\int_{0}^{\infty} t^{-1} J_{0}(t) d t \\
& \quad=2 z^{-1} \sum_{k=0}^{\infty}(2 k+3)[\psi(k+2)-\psi(1)] J_{2 k+3}(z) \\
& \quad=1-2 z^{-1} J_{1}(z)+2 z^{-1} \sum_{k=0}^{\infty}(2 k+5)[\psi(k+3)-\psi(1)-1] J_{2 k+5}(z) .
\end{aligned}
$$

For tables of this integral when $z$ is real, see [13].

We now derive yet another expression for the exponential integral. It is known $[14,15,16]^{*}$ that

* We take this occasion to point out some typographical errors in [15] and [16]. The formula $\left(\partial J_{\nu}(z) / \partial v\right)_{v=k}$ should read

$$
\left.\frac{\partial J_{\nu}(z)}{\partial \nu}\right|_{\nu=k}=-G_{k}(z)+\frac{1}{2} k ! \sum_{n=0}^{k-1} \frac{\left(\frac{1}{2} z\right)^{n-k} J_{n}(z)}{n !(k-n)}, \quad G_{k}(z)=-\frac{\pi}{2} Y_{k}(z) .
$$




$$
E i(-2 z)=(\pi z / 2)^{1 / 2} e^{-z}\left[\left.\frac{\partial I_{\nu}(z)}{\partial_{\nu}}\right|_{\nu=1 / 2}+\left.\frac{\partial I_{\nu}(z)}{\partial_{\nu}}\right|_{\nu=-1 / 2}\right]
$$

where $E i(-z)$ is given by (3.6). To evaluate the partial derivatives in (6.8), we use the expansion [17]

$$
I_{\nu}(z)=\frac{(z / 2)^{\nu}}{\Gamma(\nu)} \sum_{k=0}^{\infty} \frac{(-1)^{k} z^{k} I_{k}(z)}{(\nu+k) k !},
$$

and find that

$$
\begin{gathered}
\int_{0}^{z} t^{-1}\left(1-e^{-t}\right) d t=2 e^{-z / 2} \cosh z / 2+z g_{1}(z)-2 g_{2}(z), \\
e^{-z} \int_{0}^{z} t^{-1}\left(e^{t}-1\right) d t=-2 e^{-z / 2} \cosh z / 2+z g_{1}(z)+2 g_{2}(z),
\end{gathered}
$$

where

$$
g_{m}(z)=\sum_{k=0}^{\infty} \frac{(-1)^{k}(z / 4)^{k} e^{-z / 2} I_{k}(z / 2)}{k !(2 k+3-2 m)^{2}}
$$$$
m=1,2
$$

Of all the exponential integral expressions given, this converges the best.

\section{Numerical Examples.*}

EXAMPLE 1. Using (1.8) and (1.9), we illustrate the computation of

$$
\begin{gathered}
e^{-i x} \Phi(m+1-i a, 2 m+2,2 i x)=\sum_{k=0}^{\infty} \epsilon_{k} R_{k} i^{k} J_{k}(x), \\
R_{k}={ }_{3} F_{2}\left(-k, k, m+1+i a ; 2 m+2, \frac{1}{2} ; 1\right) .
\end{gathered}
$$

Suppose $a=1, m=2$. Then values of $i^{k} R_{k}$ are easily generated using (7.2) and (1.20). We have $R_{0}=1, i R_{1}=\frac{1}{3}, i^{2} R_{2}=\frac{1}{2} \frac{9}{1}$, etc. Using standard tables of Bessel functions, if $x=1$, seven terms of (7.1) give the six-decimal value 1.451140 . If $x=2$, nine terms give 1.293748. For tables of (7.1), see [18]. If $x=2$, about 15 terms of the Taylor series are required to achieve six-decimal accuracy.

EXAMPLE 2. We employ (2.7) and (2.8) to compute the error integrals for $z=2$. Using nine terms, we find Erf $2=0.8820814$ and $e^{-4} \operatorname{Erfi} 2=0.3013404$. About 19 terms of the Taylor series expansion are needed for the same accuracy.

EXAMPLE 3. To illustrate computation of the exponential integral, it is sufficient to consider (6.10). If $z=4$ and we use six terms of each series in (6.12), then the value of the integral (6.10) is 1.9672894 . Similarly, if $z=8$, only eight terms of each series in (6.12) are needed to yield the value 2.65669 49. For $z=8$, about 32 terms of the Taylor series expansion would be required to achieve the same accuracy.

* The author acknowledges the assistance of Sue Chapman and Betty Kahn for the development of numerical examples. 
Midwest Research Institute,

Kansas City, Missouri

1. H. Buchноцz, Die Konfluente Hypergeometrische Funktionen, Springer, 1953, p. 130.

2. F. Tricomi, "Sul comportamento asintotico dei polinomi di Laguerre", Annali di Matematica Pura ed Applicata (4), v. 28, 1949, p. 263-289.

3. F. Tricomi, "Sulla funzione gamma incompleta", Annali di Matematica Pura ed Applicata (4), v. 31,1950 , p. 263-279.

4. I. Stegun \& M. Abramowitz, "Generation of Bessel functions on high speed computers", MTAC, v. 11, Oct., 1957, p. 255-257.

5. A. Erdelyi, W. Magnus, F. Oberhettinger, \& F. G. Tricomi, Higher Transcendental Functions, McGraw-Hill Book Co., Inc., 1953, v. 1, Ch. 6. For short, in the sequel we write H.T.F., etc.

6. G. N. Watson, A Treatise on the Theory of Bessel Functions, Cambridge University Press, 1945, p.'22. Also H.T.F., v. 2, p. 7.

7. H.T.F., v. 1, Ch. 2 and Ch. 4.

8. H.T.F., v. 1, p. 80.

9. See 6, p. 139 .

10. H.T.F., v. 1 , Ch. 1

11. H.T.F., v. 1, p. 276-277. See also 2 and 3.

12. H. T. F., v. 2 , p. 148 .

13. A. N. Lowan, G. Blanch, \& M. Abramowitz, "Table of $J i_{0}(x)=\int_{x}^{\infty} \frac{J_{0}(t)}{t} d t$ and related functions", in Tables of Functions and Zeros of Functions, National Bureau of Standards, AMS 37, November, 1954, p. 33-39.

14. P. R. Ansell, \& R. A. Fisher, "Note on the numerical evaluation of a Bessel function derivative", London Math. Soc. Proc., v. 24, 1926, p. liv-lvi.

15. J. AIREY, "The Bessel function derivative $\partial J_{\nu}(x) / \partial \nu$ and $\partial^{2} J_{\nu}(x) / \partial \nu^{2}$ ", Phil. Mag., v. 19, 1935, p. 236-243.

16. F. Oberhettinger, "On the derivative of Bessel functions with respect to the order", Jour. Math. \& Phys., v. 37, 1958, p. 75-78.

17. See 6, p. 143.

18. A. N. Lowan \& W. Horenstein, "On the function $H(m, a, x)=\exp (-i x) F(m+1-$ $\imath a, 2 m+2 ; 2 i x)$ '?, in Tables of Functions and of Zeros of Functions, National Bureau of Standards, AMS 37, November, 1954, p. 1-20. 